\title{
Focal magnetic resonance guided focused ultrasound for prostate cancer: Initial North American experience
}

\author{
Uri Lindner, MD, ${ }^{*}$ Sangeet Ghai, MD; ${ }^{\dagger}$ Paula Spensieri, RN; ${ }^{\dagger}$ Eugen Hlasny, MT (MRT); ${ }^{\dagger}$ \\ Theodorus H. Van der Kwast, MD,s Stuart A. McCluskey, MD, ${ }^{\neq}$Masoom A. Haider, MD; ${ }^{+}$ \\ Walter Kucharczyk, MD; John Trachtenberg, MD, FRCSC*
}

*Division of Urology, Department of Surgical Oncology, University Health Network, and Department of Surgery, University of Toronto, Toronto, ON; 'Department of Medical Imaging, University Health Network, Toronto, ON; §Department of Pathology, University Health Network, Toronto, ON; ‘Department of Anesthesia and Pain Management, University Health Network, Toronto, ON

Cite as: Can Urol Assoc J 2012;6(6):E283-E286. http://dx.doi.org/10.5489/cuaj.12218

\section{Abstract}

The treatment of low-risk prostate cancer is a common clinical dilemma between standard curative whole gland therapy (and its associated quality of life diminishing side effects) and active surveillance (and its low, but real, risk of progression). The goal of focal therapy in low-risk prostate cancer is to achieve the best balance between cancer control and maintenance of quality of life. Magnetic resonance-guided focused ultrasound (MRgFUS) surgery is a non-invasive thermal ablation method that integrates magnetic resonance imaging for target identification, treatment planning and closed-loop control of thermal deposition and focused ultrasound for thermal ablation of the tumour target. This novel transrectal system allows for tumour localization, targeting and monitoring of tumour target ablation in real time, while simultaneously preserving adjacent normal tissue thereby minimizing the side effects of standard curative surgical or radiation therapy. We report the first North American clinical experience of treatment of localized prostate cancer with focal MR-guided transrectal focused ultrasound (clinicaltrial.gov identifier NCT01226576).

\section{Introduction}

With increased prostate cancer awareness and screening, most men currently diagnosed with this disease fall into the low-risk category with minimal risk of disease progression. ${ }^{1}$ Standard curative therapies have traditionally included radical prostatectomy and whole-gland radiation therapy (external beam or brachytherapy). These procedures, although clinically effective, incur a significant risk of quality of life diminishing morbidity, including incontinence, impotence and rectal injury. ${ }^{2}$ However, a variety of clinical trials have demonstrated that surveillance of men with low-risk prostate cancer with delayed treatment when necessary (also known as active surveillance [AS]) is associated with a minimal risk of disease-specific mortality and delays or elimi- nates the risk of side effects. ${ }^{3}$ However, most men currently diagnosed with low-risk disease in the United States have chosen curative therapy rather than risk the specter of a prostate cancer death. ${ }^{4}$

The desire to achieve oncological control with minimal side effects has driven research into minimally invasive focal procedures designed to eliminate the aggressive portions of the tumour without affecting surrounding critical structures. ${ }^{5}$ Focal high-intensity focused ultrasound (HIFU) therapy is a non-invasive option that has been used for localized prostate cancer therapy since the 1990s. Magnetic resonance imaging (MRI) of the prostate is considered the most accurate imaging technique to detect higher grade, higher risk prostate cancer. ${ }^{6}$ MRI integration with HIFU (or magnetic resonance-guided focused ultrasound [MRgFUS]) uses the MR images for accurate localization and for targeting the tumour for ablation. Furthermore, the phase shift characteristics associated with echo planer sequence MRI allow for accurate near real time temperature determination (MR thermography) of the treated zone and surrounding normal tissue.

We report the feasibility and safety of focal transrectal MRgFUS on the first North American patient treated for localized prostate cancer using a transrectal device (ExAblate 2100, Insightec Inc., Haifa, Israel). The ExAblate system provides a real time therapy planning algorithm, thermal dosimetry and closed loop therapy control. The physician initiates therapy by acquiring MR images of the prostate area to be ablated and drawing treatment contours. The therapy planning software automatically computes the type and number of sonifications required to treat the defined treatment area, while minimizing total treatment time. MR images taken during the actual sonifications provide quality diagnostic images of the target tissue and a qualitative near real time map overlay to confirm the therapeutic thermal effect of the treatment of each volume. The focus is then automatically moved electronically to the succeeding treatment adjacent point and the process is repeated until the entire treatment volume is ablated. 
One other study of MRgFUS on canine and human patients has been published, but the human feasibility study used a transurethral device and targeted only the central prostate without a specific cancer target. ${ }^{7}$ There have been several reports at clinical meetings of non-North American patients treated with ExAblate MRgFUS, but no peer-reviewed publications.

\section{Case report}

A 51-year-old patient, with a palpable right prostatic nodule on digital rectal exam (clinical stage $\mathrm{T} 2 \mathrm{a}$, prostate-specific antigen of $1.07 \mathrm{ng} / \mathrm{mL}$ ) and low-risk Gleason 6 cancer on previous biopsy, consented and enrolled in this Institutional Research and Ethics Board (IRB) approved study. Validated baseline sexual (International Index of Erectile Function [IIEF]), urinary (International Prostate Symptom Score [IPSS]), and quality of life (Patient Oriented Prostate Utility Scale [PORPUS]) data were obtained.

The patient underwent multiparametric 3T MRI (Siemens, MAGNETOM, Verio 3T) with an endorectal coil, followed by a confirmatory transrectal ultrasound (TRUS)-guided extended (16 cores) mapping biopsy (regular 12 cores +2 peri-urethral samples in each half of the gland at the level of base and the mid-gland). The proximal end of each biopsy sample was inked to allow us to identify the location of the tumour. Biopsy results reconfirmed Gleason $6(3+3)$ cancer in right mid-lateral ( $1 \%$ of 1 core) and right medial samples at the level of the mid-gland ( $1 \%$ of 1 core). Template mapped trans-perineal biopsies were not performed because the IRB was reluctant to introduce a non-local standard of care procedure for what was considered a low-risk disease. Since the tumour was not visible on MR images, the treatment target volume included the two sectors from which the cores were positive. A computed tomography of the pelvis ruled out calcification around the rectum in the expected beam path. MRgFUS treatment was performed under general anesthesia as an outpatient procedure, one month after the biopsy. The procedure was carried out on a 1.5T GE Excite Twinspeed MR scanner (GE Healthcare, Waukesha, WI) using the ExAblate 2100 prostate system (InSightec Ltd., Haifa, Israel). When the patient was placed in a low lithotomy position in a custom MR gantry, a urethral catheter was inserted at the beginning of the procedure. The ExAblate 2100 system was positioned transrectally. T2 weighted images were obtained before the ablation to verify adequate coverage of the prostate by the device. Prostate boundaries, rectum, urethra and neurovascular bundle, as well as the area targeted for ablation, were traced on the MR images (Fig. 1).

The HIFU device allows for a transrectal thermal delivery, while cooling the rectal mucosa with closed loop cold circulating water system. Five macro-sonications were required to treat the area targeted for the prescribed cancer therapy. The ablation time for each macro-sonication was $160 \mathrm{sec}-$

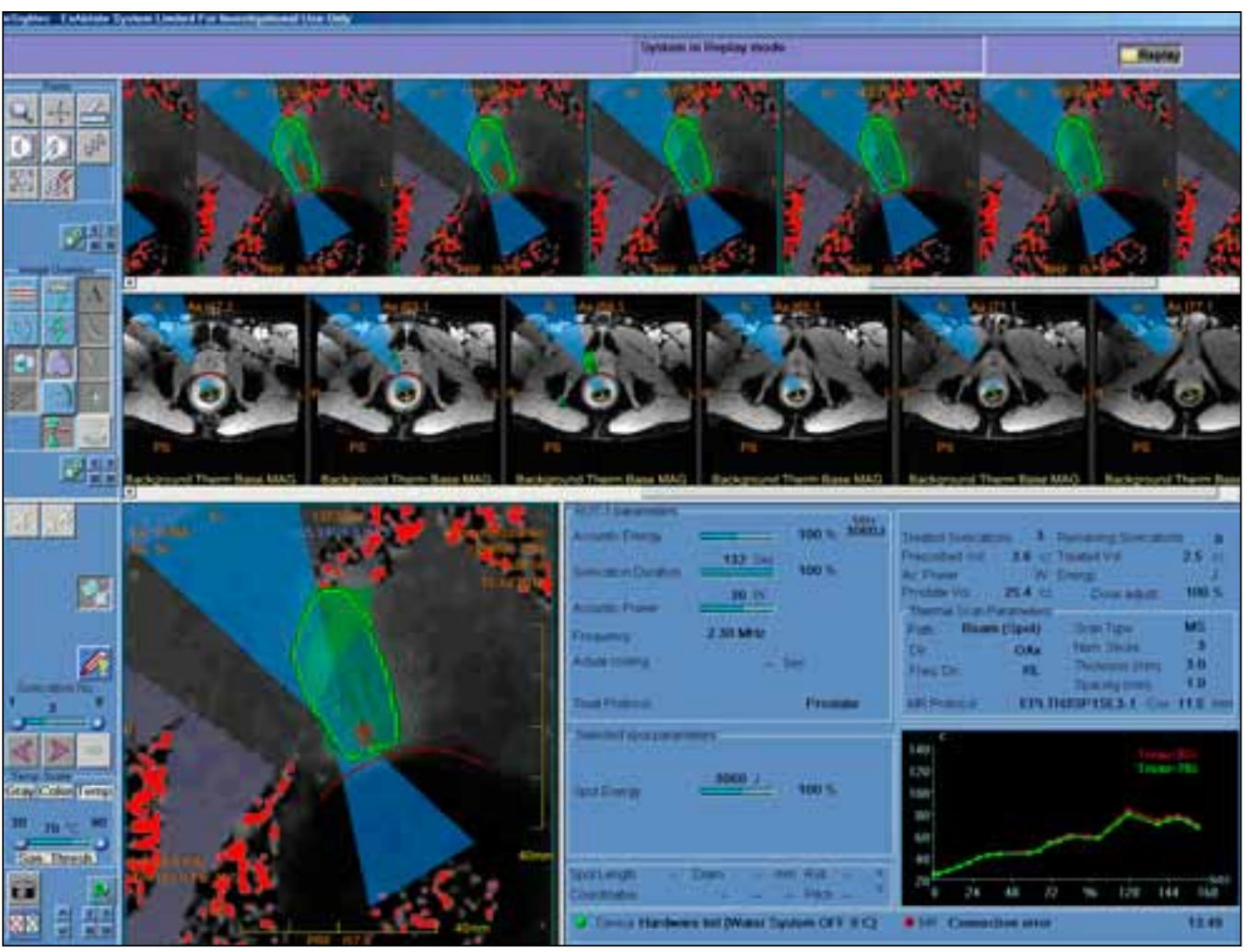

Fig. 1. Intra-procedure image of the prostate, including the tumour registration. 
onds. An additional regular sonication was used at the end to cover a marginal dose region at the lateral aspect of the treated region. Each sonication was monitored by MR thermometry that calculates tissue temperature and extrapolates cell death based on validated prostate Ahrenius calculations. Similarly, the thermography showed no significant heating of the areas expected to be preserved. The patient was in the scanner for a total of 3.5 hours, of which the treatment time was about 70 minutes. The final non-perfused volume (NPV) at the end of the treatment was $3.8 \mathrm{cc}$ (Fig. 2). The patient was transferred to the postoperative recovery unit; the catheter was removed and he was discharged the same day voiding spontaneously.

The procedure was uneventful and no intra-procedural complications were noted. At the 1 week and 1 month follow-up visits after the procedure, the patient did not experience any unpleasant side effects, did not use any pain medication, and was back to his regular active lifestyle within a few days of the ablation. There were no significant changes in IIEF-15, IPSS (Fig. 3) and PORPUS at these visits. Repeat $\mathrm{MRI}$ at 1 month showed persistent ablated non-perfused tissue at the right mid-gland (Fig. 4).

\section{Discussion}

The primary goal of this phase 1 study was to evaluate the safety of focal MRgFUS treatment of locally confined lowrisk prostate cancer. Patients for this study were selected in accordance with the focal prostate cancer consensus panel, ${ }^{5}$

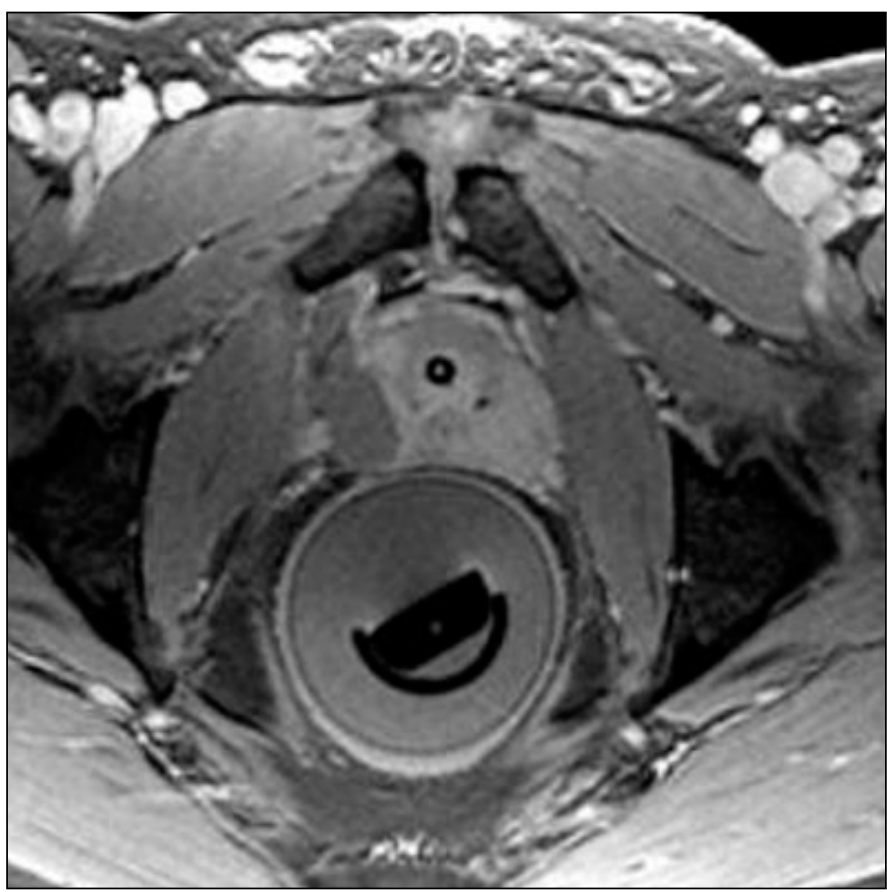

Fig. 2. Post-contrast magnetic resonance image obtained immediately after treatment showing a non-perfusion area in the right gland at the treatment site. however, we excluded patients with more aggressive disease with a combination of two transrectal guided biopsies and multiparametric MRI as opposed to the proposed transperineal mapping biopsy. Our combination of investigations biases the discovery of high-grade tumours at higher risk of progression at the expense of missing elements of low-grade tumours with low risk of progression; these low-risk patients would have undergone unnecessary radical intervention and subsequent side effects. Since the consensus panel agreed that advanced imaging should play a major role in the detection of high-grade cancer and performance of FT, we felt that the combination of a careful TRUS biopsy and a complimentary high resolution multiparametric MR imaging would be sufficient for the purpose of eliminating higher risk disease (though not necessarily all low-risk cancer) in our carefully selected patient population. ${ }^{6,7}$

Whole gland HIFU is associated with urinary retention ( $1 \%$ to $20 \%$ ), urinary tract infections $(1.8 \%$ to $47.9 \%)$, stress or urinary incontinence $(<1 \%-34.3 \%)$, erectile dysfunction $(20 \%-81.6 \%)$ and recto-urethral fistulae $(<2 \%)$. It also necessitates the insertion of a supra-pubic catheter for varied periods of time. ${ }^{8}$ Even non-MR-guided focal HIFU is associated with dysuria $(22 \%)$, urethral sloughing $(34 \%)$, urinary tract infection $(17 \%)$, and necessitates insertion of a supra-pubic catheter. ${ }^{9}$

In our first case, we have demonstrated that this procedure can be performed safely with no adverse events up to 1 month post-procedure. It also demonstrates that we were successful in effectively devascularizing the area that we targeted, with persistent non-perfusion at the site of ablation at the 1 month follow-up scan (Fig. 4). We believe that added complexity of using MRI-guided FUS over ultrasoundguided FUS is well worth it for the precision and uniformity of tumour devascularization, as well as the ability to avoid unwanted tissue damage by monitoring temperatures in the treatment field. The short- and long-term oncologic outcome

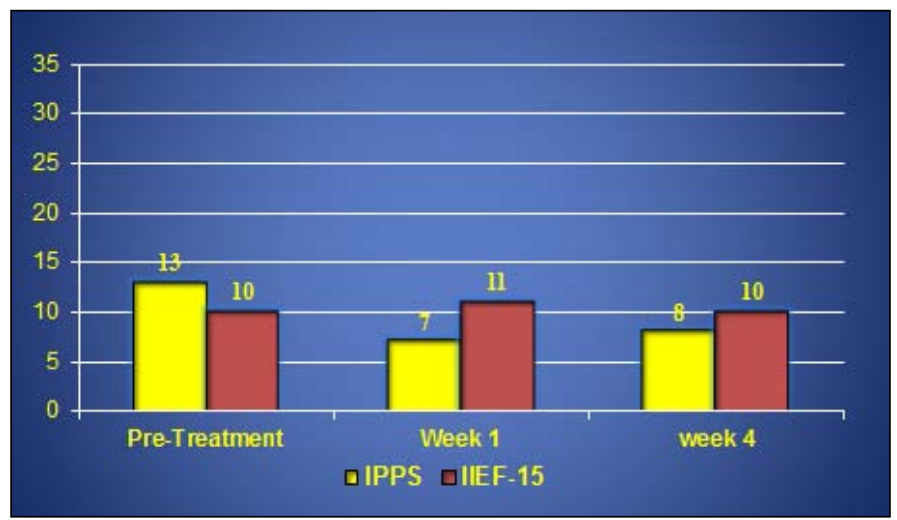

Fig. 3. Changes in voiding and sexual function after magnetic resonance guided focused ultrasound. There were no significant changes in voiding and sexual function as measured by the International Prostate Symptom Score (IPSS) and International Index of Erectile Function (IIEF)-15 from baseline to one week and one month. 


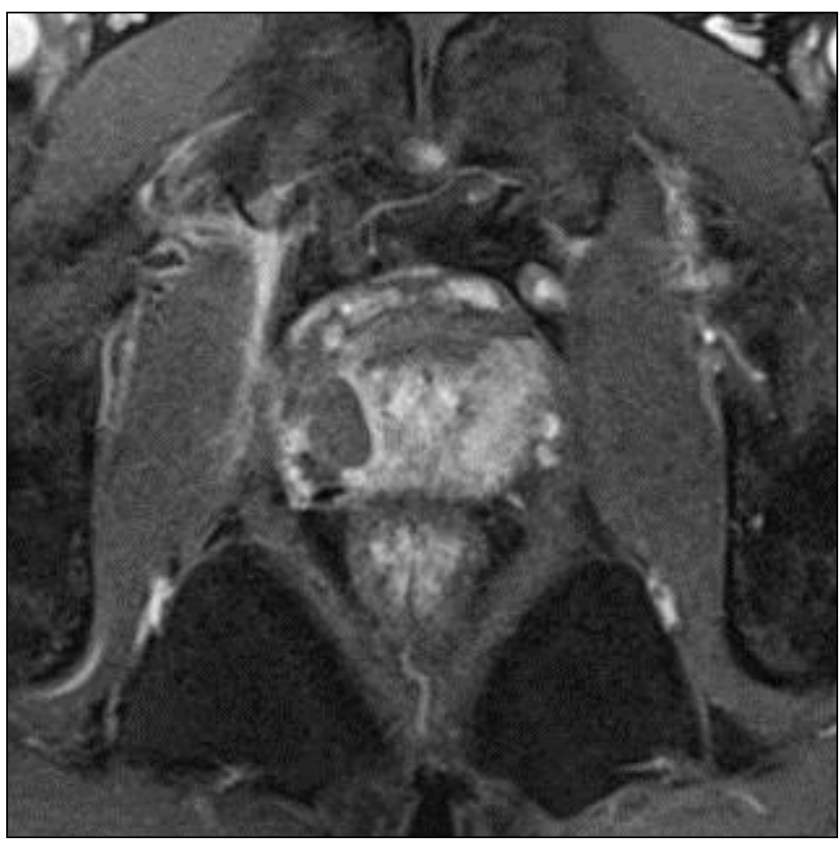

Fig. 4. One month post-treatment, the magnetic resonance scan following contrast enhancement shows persistent area of non-perfusion in treated right aspect of the prostate.

remains to be determined by sequential biopsies. The present patient awaits repeat biopsy, which will be performed at 6 months post-ablation, as per protocol.

\section{Conclusion}

This is the first reported North American experience with transrectal MRgFUS for the treatment of localized PCa. This case report affirms the feasibility of performing this procedure in humans. Further studies are ongoing to document safety in a larger cohort of men and to document early oncologic outcome.
Competing interests: None declared. National Clinical Trial Registry \#NCT01094665

Acknowledgements: The authors would like to thank InSightec Ltd. for supporting this study, the Ontario Research Fund (ORF), and the Canadian Foundation for Innovation (CFI).

This paper has been peer-reviewed.

\section{References}

1. Rider JR, Sandin F, Andrén 0, et al. Long-term Outcomes Among Noncuratively Treated Men According to Prostate Cancer Risk Category in a Nationwide, Population-based Study. Eur Urol 2012, Epub Aug 10. http://dx.doi.org/10.1016/i.eururo.2012.08.001

2. Sanda MG, Dunn RL, Michalski J, et al. Quality of life and satisfaction with outcome among prostatecancer survivors. N Engl J Med 2008;358:1250-61. http://dx.doi.org/10.1056/NEJMoa074311

3. Klotz L, Zhang L, Lam A, et al. Clinical results of long-term follow-up of a large, active surveillance cohort with localized prostate cancer. J Clin Oncol 2010;28:126-31. http://dx.doi.org/10.1200/ JC0.2009.24.2180

4. Whitson JM, Porten SP, Carroll PR. Prostate cancer: reducing overtreatment: active surveillance in low-risk disease. Nat Rev Urol 2011;8:124-5. http://dx.doi.org/10.1038/nrurol.2011.8

5. de la Rosette J, Ahmed H, Barentsz J, et al. Focal therapy in prostate cancer-report from a consensus panel. J Endourol 2010;24:775-80. http://dx.doi.org/10.1089/end.2009.0596

6. Nagarajan R, Margolis D, Raman S, et al. Correlation of Gleason scores with diffusion-weighted imaging findings of prostate cancer. Adv Urol 2012;2012:374805.

7. Siddiqui K, Chopra R, Vedula $S$, et al. MRl-guided transurethral ultrasound therapy of the prostate gland using real-time thermal mapping: initial studies. Urology 2010;76:1506-11. http://dx.doi. org/10.1016/i.urology.2010.04.046

8. Cordeiro ER, Cathelineau $X$, Thuroff $S$, et al. High-intensity focused ultrasound (HIFU) for definitive treatment of prostate cancer. BJU Int 2012, Epub Jun 6.

9. Ahmed HU, Hindley RG, Dickinson L, et al. Focal therapy for localized unifocal and multifocal prostate cancer: a prospective development study. Lancet Oncol 2012;13:622-32. http://dx.doi.org/10.1016/ S1470-2045(12)70121-3

Correspondence: Dr. John Trachtenberg, University Health Network, Princess Margaret Hospital, Room 3-208, 610 University Ave., Toronto, ON M5G 2M9; fax: 416-946-5558; john.trachtenberg@utoronto.ca 